\title{
A first look at the IP eXchange Ecosystem
}

\author{
Andra Lutu \\ Telefonica Research \\ andra.lutu@telefonica.com \\ Diego Perino \\ Telefonica Research \\ diego.perino@telefonica.com
}

\author{
Byunjin Jun \\ Northwestern University \\ byungjinjun2022@u. \\ northwestern.edu
Marcelo Bagnulo
University Carlos III of Madrid
marcelo@it.uc3m.es

\author{
Fabián E. Bustamante \\ Northwestern University \\ fabianb@cs.northwestern.edu
}

\section{Carlos Gamboa Bontje Telefonica \\ carlos.gamboabontje@telefonica.}

\begin{abstract}
The IPX Network interconnects about 800 Mobile Network Operators (MNOs) worldwide and a range of other service providers (such as cloud and content providers). It forms the core that enables global data roaming while supporting emerging applications, from VoLTE and video streaming to IoT verticals. This paper presents the first characterization of this, so-far opaque, IPX ecosystem and a first-of-its-kind in-depth analysis of an IPX Provider (IPX-P). The IPX Network is a private network formed by a small set of tightly interconnected IPX-Ps. We analyze an operational dataset from a large IPX-P that includes BGP data as well as statistics from signaling. We shed light on the structure of the IPX Network as well as on the temporal, structural and geographic features of the IPX traffic. Our results are a first step in understanding the IPX Network at its core, key to fully understand the global mobile Internet.
\end{abstract}

\section{CCS CONCEPTS}

- Networks $\rightarrow$ Mobile networks; Network measurement; Topology analysis and generation;

\section{KEYWORDS}

Mobile Networks, Roaming, IPX Network, Interconnection

\section{INTRODUCTION}

International roaming is an important feature of cellular networks, allowing subscribers to use their devices anywhere in the world as if at home. Under the IP Packet Exchange (IPX) model [5, 6], MNOs contract the services of third party providers - the IPX Provider (IPX-P) - to offer their customers access to mobile services in any foreign country. No IPX-P on its own is able to provide connections on a global basis (e.g., single-handily interworking with all MNOs). Thus, IPX-Ps peer to other IPX-Ps [18] to expand their geographical footprint. The resulting IPX Network, is an isolated network that bypasses the public Internet [3], to

ACM SIGCOMM Computer Communication Review

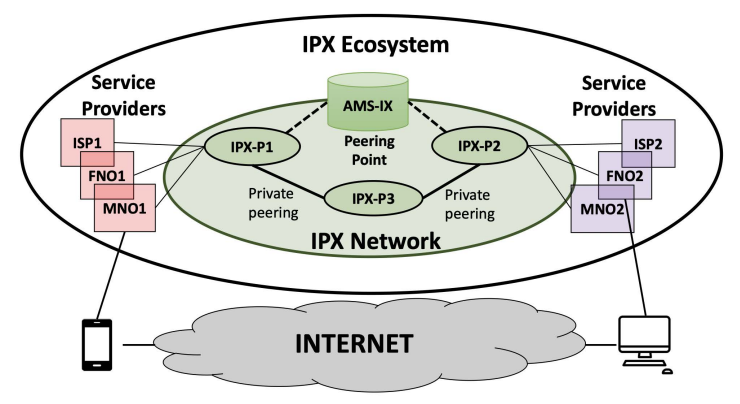

Figure 1: High level architecture of the IPX Ecosystem.

ensure secure, SLA-compliant services, from video streaming and AR/VR to IoT verticals, such as connected cars.

Recent years have brought a rapid growth in the number of participants in the IPX ecosystem and the volume of traffic they exchanged. The growing number of international travelers, reaching 1.4 billion in 2018 [1] and the "flat-rating" or elimination of international roaming charges $[8,9,19]$ has led to an exponential growth in roaming traffic, expected to increase 32-times by 2022. At the same time, users' QoE expectations - when using VoIP or posting videos - has forced content and service providers to peer close to their users, wherever they may roam, thus adding to a growing interconnection ecosystem.

Despite its rapid growth and increased importance as the core of the mobile Internet, the IPX Network and its associated ecosystem (Figure 1) has received little to no attention by our community, due in part to its intrinsic opacity and separation from the public Internet. In this paper, we present the first characterization of the IPX ecosystem and a first-ofits-kind, detailed study of a large operational IPX Provider.

We contribute the first topology analysis of the IPX ecosystem. We analyze a private BGP routing table snapshot from an operational router that is part of the IPX Network to map the interconnection between IPX-Ps and Service Providers (SPs) for the data roaming service (§ 3). We build the exhaustive list of 29 active IPX-Ps and detail their approach for

Volume 50 Issue 4, October 2020 
peering using three major peering exchange points (AMSIX Amsterdam, Equinix Ashburn and Equinix Singapore). ${ }^{1}$ We capture the breadth and full-mesh peering fabric of the IPX Network for data roaming, which enables inter-working between all $\approx 800$ MNOs currently active world-wide.

We present a first-of-its-kind detailed analysis of a large operational IPX-P system, and provide the insider view into the otherwise inaccessible IPX Network. Our study is based on real-world traffic records for its main service (radio signaling for data roaming (§ 4)) over a two-week period in December 2019. We showcase the operational IPX-P's signaling and data roaming infrastructures ( $§ 4.2)$, with notable presence in Europe and the Americas. We study signaling traffic patterns (for different radio access technology) and data communications from over 22 million mobile devices roaming in the world.

\section{THE IP EXCHANGE MODEL}

In this section, we provide a detailed description of IPX and use data roaming, one of the main services offered, to illustrate the main IPX players and their interactions.

\subsection{IPX Connectivity for SPs}

At a high-level, the IPX ecosystem (Figure 1) includes Service Providers (SP) and networked IPX-Providers (IPX-Ps). IPX-Ps are third-party interconnection providers to SPs (e.g., MNOs, Internet of Things (IoT) providers). IPX-Ps peer with other IPX-Ps to extend their footprint worldwide.

While IP-based, the resulting IPX Network is a private network, separate from the public Internet, that meshes together the infrastructures of the IPX-Ps. It guarantees traffic separation between IPX services from the rest of the Internet. The IPX Network enables the transport of global roaming data between networks, with interoperability of different implementations and standards.

SPs require a single connection and agreement with one IPX-P in order to connect to the IPX Network, and interconnect with partner SPs world-wide. ${ }^{2}$ For instance, to enable data roaming, two MNOs must each have an agreement with an IPX-P in order to interconnect. For redundancy, a SP could establish connections to more than one IPX-P. Depending on the footprint of the IPX-P's infrastructure, SPs can use one or more Point of Presences (PoPs) of the IPX-P.

\footnotetext{
${ }^{1}$ IPX is not the same as IXP, though the acronyms are similar. IPX-Ps may rely on IXPs (such as AMS-IX) for peering with other IPX-Ps.

${ }^{2}$ Although direct interconnection between SPs through leased lines or Virtual Private Network (VPN) is possible, it is outside the scope of our analysis.
}

ACM SIGCOMM Computer Communication Review

\subsection{Study Case: Data Roaming over IPX}

To establish roaming, roaming partner MNOs must have a functioning commercial agreement, implement their roaming technical solutions, establish inter-working and deploy their billing function. We use data roaming to illustrate the main IPX ecosystem players and their interactions.

In terms of business agreement solutions, the legacy option for MNOs is a standard bilateral agreement where the two parties involved define terms and conditions of their cooperation. These bilateral roaming agreements for roaming and inter-working are costly and generally of lower value today, something that has served as additional motivation for MNOs to adopt the IPX model.

Under the IPX model, operators connect to an IPX-P to gain access to many roaming partners world-wide, externalizing the inter-working establishment to the IPX-P offering the service. IPX-Ps are then peering with each-other to expand their international footprint through the IPX Network. This IPX hubbing solution does not preclude the existence of bilateral agreements between MNOs, which can be viewed as a complementary roaming model.

Once a commercial agreement has been created, the IPX-P sets up the technical roaming solution, including coordination over the signaling platform, and establishes the IPX-P interconnectivity. After MNOs establish roaming inter-working, they deploy the billing service, which is key to recovering roaming revenue. The roaming partners must each record the activity of roaming users in a given Visited Mobile Network Operator (VMNO). Then, by exchanging and comparing these records, the VMNO can claim revenue from the partner Home Mobile Network Operator (HMNO).

When a mobile device is at home, the subscriber's traffic will take a short path inside the network to reach a suitable Packet Data Network Gateway (PGW) to the Internet. When inter-working exists between two MNOs, there are several network configurations the IPX Network supports to enable roaming. The IPX-P's main function is to build the communication tunnel between the Serving Gateway (SGW) and the $P G W$, enabling traffic to flow to and from the roaming mobile device. The traffic of a roaming mobile device is directed to an egress PGW whose location depends on the roaming configuration. Different configurations for roaming over the IPX Network are available - home-routed roaming (HR), local breakout (LBO) and IPX hub breakout (IHBO). Prior work found that the default roaming configuration majority MNOs currently use in Europe is the HR roaming [15]. In the case of $\mathrm{HR}$, the mobile device receives the IP address from its home $\mathrm{MNO}$ and the roaming traffic is then routed over a tunnel between the SGW in the VMNO and a PGW in the HMNO.

Volume 50 Issue 4, October 2020 


\section{IPX ECOSYSTEM DIVERSITY}

The core players of the IPX ecosystem are IPX-Ps and SPs. IPX-Ps provide the interconnection between SPs directly through their network or via peering with other IPX-Ps (see Figure 1). The resulting ecosystem has a layered topology: a core of tightly interconnected IPX-Ps in a full mesh (the IPX Network) and the edge of diverse SPs that interconnect through the IPX Network, either through a single IPX-P or multi-homed through multiple IPX-Ps. For this, SPs may use their own access network (e.g., fixed and mobile network operators) or use a local provider to connect to the PoP of the IPX-P (e.g., Application Service Providers (ASP)). SPs originate and/or terminate traffic for one or several services; they do not transport traffic.

\subsection{IPX-Ps Interconnection and Peering}

IPX-Ps establish interconnection either through private bilateral interconnections or through an Internet Exchange Point (IXP) (Figure 1). The benefits of peering are well known among Internet Service Providers (ISPs) and Content Delivery Networks (CDNs), particularly when it comes to public peering via an IXP [2]. In recent years, we have seen more efforts to expand the peering culture to the mobile ecosystem [18], by establishing new mobile peering infrastructure world-wide. This is (slowly) propagating to the mobile industry, and the interconnection of IPX-Ps via mobile peering at specific IXPs, which offer this service, is becoming commonplace. Currently, the two major IXPs offering the mobile peering service are AMS-IX and Equinix, with three locations overall (Amsterdam, Ashburn and Singapore).

IPX-Ps dynamically exchange routing information with other IPX-Ps using the BGP routing protocol. According to GSMA recommendations [6], IPX-Ps should not act as a transit IPX-P (i.e., there can only be a maximum of two IPXPs between two partner SPs). Therefore, when an IPX-P has a customer SP who requires a connection to a customer SP of another IPX-P, the two IPX-Ps should peer, either through (direct) private peering or peering points. For example, in Figure 1, the path between MNO1 and MNO2 should only traverse two IPX-Ps (i.e., MNO1 $\rightarrow$ IPX-P1 $\rightarrow$ IPX-P2 $\rightarrow$ MNO2). Also, IPX-P3 should never transit traffic for neither of its two peers, IPX-P1 and IPX-P2. To ensure this, network routes IPX-P3 receives either over private peering or over a peering point should not be re-advertised to other IPX-P peering partners. These recommendations result in a tightly interconnected IPX Network, with a theoretical diameter of two entities between any pair of SPs.

\subsection{IPX Topology}

Given the opaque nature of the IPX ecosystem, we cannot capture its characteristics from the public Internet, not by

ACM SIGCOMM Computer Communication Review

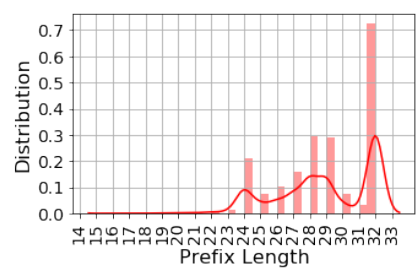

(a) IPv4 prefix length.

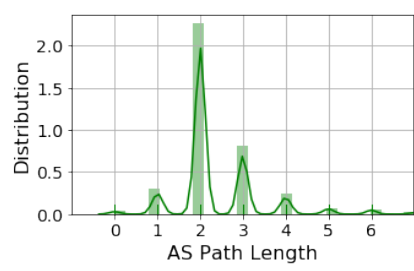

(b) AS-Path length.
Figure 2: Analysis of the prefixes within the routing table snapshot from an operational IPX-P: (a) distribution on prefix length; (b) distribution on AS-Path length (without ASPath prepending).

using public Internet routing data nor with active end-to-end measurements (e.g., traceroute). In order to shed light on the interconnection fabric between IPX-Ps and SPs in the IPX ecosystem, we analyze three different datasets: (i) routing dataset: a private BGP routing table snapshot for data roaming that one of the largest operational IPX-Ps provided us; (ii) peering dataset: the list of AMS-IX members that connect to the mobile peering services for data roaming service only, together with the full internal list of IPX-P peers from the operational IPX-P providing us the routing dataset; (iii) survey dataset: market surveys and reports $[13,17]$ from third parties. In the routing data, we capture one snapshot of the routing table on the 30th of January 2020 , which provides one view of the IPX-P's relationships for the data roaming service. We note that this information, though descriptive of the ecosystem, might be incomplete (i.e., there might be information not included here, but present in snapshots at other vantage points).

Specifically, the IPX-P routing table snapshot includes reachability information for a total of $10,418 \mathrm{IPv} 4$ prefixes advertised by 59 different entities (neighbors), which include the peer IPX-Ps and the customer MNOs of the IPX-P we analyze. By checking the originating AS of the prefixes in the routing dataset, we find that within the IPX ecosystem there are a total of 824 different service providers for data roaming (i.e., MNOs, MVNOs or M2M platforms). This number is consistent with the total number of MNOs active world-wide that register with the GSMA. These are public IPv4 prefixes that IPX-Ps do not announce in the global BGP routing tables. Hence, they are not reachable from the public Internet. From Figure 2a, we note that the median prefix length in the routing dataset is $/ 29$, and there is a large number of $/ 32$ prefixes that MNOs originate. These likely represent specific elements (e.g., Home Location Registry (HLR) or Mobility Management Entity (MME)) within the MNO infrastructure, which are involved in procedures for data roaming.

IPX Network: We merge and corroborate the information we extract from the above-mentioned datasets to build

Volume 50 Issue 4, October 2020 
a list of IPX-Ps that currently form the IPX Network. In Annex $C$, we detail the full list of these providers we found and the methodology we compile this list. We specifically note that, in light of the growing popularity and worldwide footprint of IPX services, many heavyweight telecoms are participating in the IPX environment, leveraging their underlying extensive infrastructure.

We verify that these IPX-Ps appear in the routing dataset as active peers of the IPX-P providing us the routing table snapshot. The total number of entities advertising reachability information to the IPX-P is equal to 59 different ASes. Out of these, we separate the ones that advertise prefixes with an AS-Path length longer than one (i.e., they advertise their SPs). We check the overlap with the list of IPX-Ps we built, and find a set of 23 different ASes. ${ }^{3}$

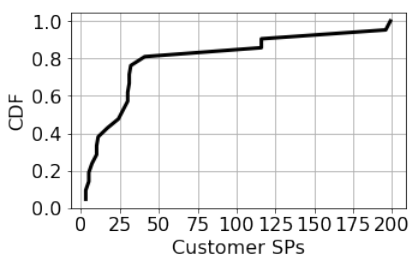

(a) Customer SPs per IPX-Ps.

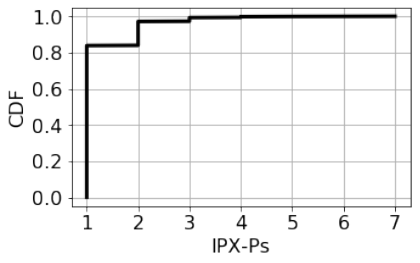

(b) IPX Providers per SP.
Figure 3: Interconnection between IPX-Ps and SPs: (a) distribution of number of customers per IPX-P; (b) distribution of number of IPX providers per SPs.

IPX Interconnections: The set of 29 IPX-Ps we found must provide interconnection services to the over $800+$ MNOs currently active world-wide [4]. The number of PoPs indicates the number of locations where an IPX-P can crossconnect with SPs, giving insight into its world-wide geographical footprint. For example, large players such as Telia Sonera, TATA Communications, Orange, Vodafone, Telecom Italia, Telefonica or Telekom Austria offer an infrastructure with more than 100 PoPs world-wide each. The average number of PoPs among the 18 IPXs publicly disclosing this information is 116 , hinting the breadth of the system we capture.

We further use the routing dataset in order to characterize the interconnection between IPX-Ps and SPs in the ecosystem. For the IPX-Ps present in the routing dataset, we verify the different number of SPs for which they advertise reachability information (Figure 3a).

We find that four major players within the IPX Network (namely, Syniverse, BICS, Orange and Comfone) together give services to a total of more than 600 MNOs (out of the total 800). Inversely, we also verify the popularity of multi-homing among SPs. In other words, we quantify the number of IPXPs that advertise reachability information for the same SP.

\footnotetext{
${ }^{3}$ The remaining 36 ASes observed in the BGP routing table are SPs that are customers of the analyzed IPX-P.
}

ACM SIGCOMM Computer Communication Review
Figure $3 \mathrm{~b}$ shows that $80 \%$ of SPs are single-homed (i.e., they only connect to one IPX-P), while for the rest we observe up to seven different IPX-Ps. In particular, we note that multiple M2M platform providers use at least four different IPX-Ps, which is intuitive due to their reliance on roaming.

Finally, in order to characterize the interconnection between IPX-Ps, we analyze the AS-Path of the prefixes in the routing dataset. To comply with the recommendation of a fully connected IPX Network, there can be no more than two different IPX-Ps involved in the communication between two different SPs. Figure $2 \mathrm{~b}$ shows the distribution of prefixes on AS-Path length. Note that we eliminated AS-Path prepending (used by IPX-Ps and SPs for traffic engineering).

We show that, indeed, majority prefixes advertised by peer IPX-Ps have a path length equal to two, confirming the tight interconnection required in the IPX Network. The paths longer than two ASes represent the result of MNOs working together with their parent networks (e.g., national MNOs connecting to their parent carrier) or third party network providers (e.g., for MVNOs, M2M platforms) to achieve a broader geographical footprint. Thus, sibling ASes that belong to the same organization appear in the same AS-Path.

We also observe that ASes in the IPX network use standard techniques for traffic engineering. In particular, we observe a heavy use of AS prepending. AS path prepending makes a route less preferred to receive traffic by making the AS path length for the route artificially long by repeating ASes in the AS path attribute. We observe 1,331 routes from 131 different origin ASes where prepending was used. We also observe extensive use of Multi Exit Discriminator (MED), a BGP attribute that serves to express preference between different links between two ASes. We observed 1,583 prefixes that contained the MED attribute.

\section{A LARGE IPX PROVIDER}

We continue our analysis of the IPX ecosystem and zoom into the operations of one of the largest IPX-Ps in the ecosystem. The IPX-P we dissect is a Tier-1 Internet Service Provider operating one of the largest backbone networks world-wide. As part of its interconnectivity products, the carrier operates an IPX infrastructure that runs on top of its vast Multiprotocol Label Switching (MPLS) network. ${ }^{4}$ The IPX-P infrastructure integrates more than 100 PoPs in 40+ countries with a particularly strong presence in America and Europe.

In terms of network connectivity, the IPX-P offers two types of interfaces, namely the IPX Access for clients (service providers) and the IPX Exchange for peering with other

\footnotetext{
${ }^{4} \mathrm{An}$ IPX-P requires access to an underlying backbone network. The IPX-P may own its own MPLS network or alternatively, it might lease capacity on MPLS networks on which they deployed the infrastructure needed to deliver and manage inter-operable cross-network services.
}

Volume 50 Issue 4, October 2020 


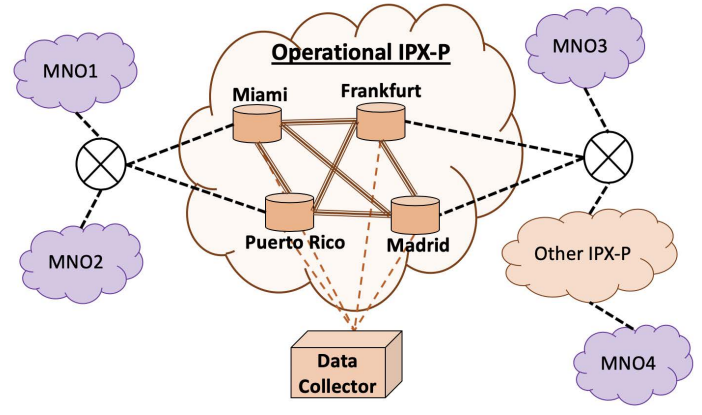

Figure 4: High level architecture of the IPX-P's signaling platform.

IPX-Ps. The main mobile peering points the IPX-P uses are those in Singapore, Ashburn and Amsterdam. By peering with other large Tier-1 carriers, the IPX-P extends its footprint world-wide to geographic regions where it does not own infrastructure (§ 3.2). The IPX-P serves clients in multiple countries in Europe (Germany, Spain, UK) and the Americas (including US, Mexico, Brazil, Argentina, Colombia, Peru, Chile, and Ecuador).

\subsection{IPX-P Infrastructure and Monitoring}

We now describe the IPX-P infrastructure, the monitoring methodology and the datasets we collected to characterize its operational system and services. We monitor the IPX-P infrastructure corresponding to the two main services - SCCP Global Signaling, LTE Diameter Exchange - for two weeks, from December 1st to December 14th 2019.

Overall, the total number of IMSIs we capture is of more than $22 M$ active daily in $2 G / 3 G$ and more than $2 M$ active daily in $4 G / L T E .^{5}$. They correspond to 215 home countries and 210 visited countries. The IPX-P's customers are active within 19 countries and include MNOs, IoT/M2M connectivity providers and cloud service providers.

SCCP Global Signaling: This service provides access to the IPX-P's SS7 signaling network, satisfying the $2 \mathrm{G} / 3 \mathrm{G}$ interconnection needs for international roaming of MNOs. The SCCP Signaling network of this particular IPX-P has a redundant configuration with four international Signaling Transfer Points (STPs) located in North America (Miami, Puerto Rico) and Europe (Frankfurt, Madrid) as depicted in Figure 4. To capture clients' activity across this signaling platform, we monitor the Mobile Application Protocol (MAP) protocol, which supports end-user mobility and is used by devices to communicate with the major network elements, including the HLR, Visiting Location Registry (VLR) or the Mobile Switching Center (MSC). We collect traffic corresponding to the following procedures of each device belonging to one of

\footnotetext{
${ }^{5}$ Note that there might be an overlap between these two sets. However, we aim to show here the load on the two different signaling infrastructures
}

ACM SIGCOMM Computer Communication Review the IPX-P's clients (outbound roaming) or to foreign devices that connect to the network of one of the IPX-P's clients (inbound roaming): i) location management (update location, update GPRS location, cancel location, purge mobile device); ii) authentication and security (send authentication information); iii) fault recovery.

LTE Diameter Exchange: This service provides the Diameter signaling capabilities necessary to enable $4 \mathrm{G}$ roaming for customers. The infrastructure of this particular IPX-P includes four Diameter Routing Agents (DRAs) meant to forward Diameter messages and simplify interworking between different network elements. The LTE Diameter service integrates value added services, including Welcome SMS, Steering of Roaming or Sponsored Roaming. To monitor the activity of the IPX-P's customers across this platform, we monitor traffic across the geo-redundant signaling network with four DRAs located two in Europe (Frankfurt, Madrid) and two in North America (Miami, Boca Raton). The infrastructure is similar to the one in Fig. 4.

\subsection{Signaling Traffic Trends}

Figure 5 shows signaling activity of roaming mobile subscribers during the observation period of December 2019. We look at both MAP and Diameter signaling procedures. MAP is the most important application protocol in the Signaling System No. 7 (SS7) stack, and handles the roamers' mobility between countries. Although this is still the most used protocol for mobile interconnection application messages, the more recent Diameter [12] signaling protocol has been growing with the adoption of LTE. Figure 5a focuses on the total signaling traffic and on the number of different mobile subscriber devices that generate this traffic.

We find that the number of devices using $2 G / 3 G$ infrastructure (extracted from MAP traffic) is an order of magnitude higher than those using $4 G$ infrastructure (based on the Diameter traffic). The volume of signaling traffic in the SCCP infrastructure is, correspondingly, more significant in terms of total volume than in the Diameter infrastructure. We also note the typical daily and weekly traffic patterns on mobile subscribers' activities. For instance, December 1st was a Sunday, showing the expected decreasing trend in signaling traffic activity which can be seen again the following weekend (December 7-8th).

Each record in this dataset represents a signaling procedure that a network element triggers, corresponding to different standard routines. For instance, from the MAP interface we capture mobility management routines, including location management and authentication. Figure $5 \mathrm{~b}$ shows the time series of signaling traffic broken down by type of signaling procedure, including Update Location (UL), Cancel Location (CL) and Send Authentication Information (SAI)

Volume 50 Issue 4, October 2020 

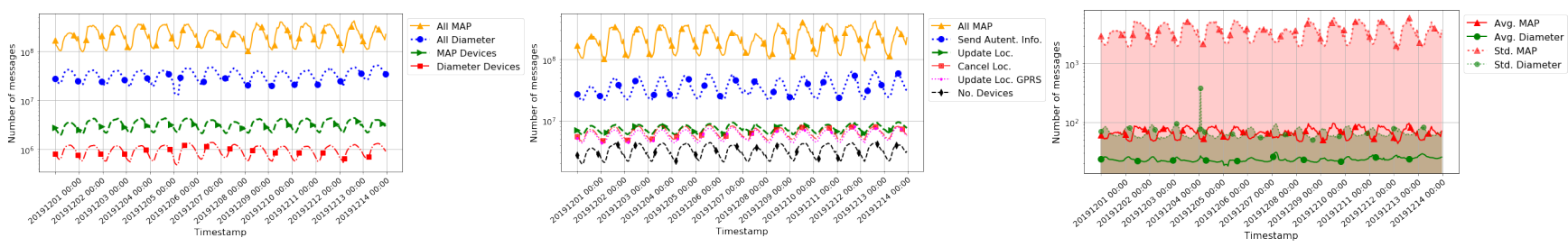

(a) Signaling traffic volume and number of devices in each(b) SCCP signaling traffic time series; breakdown per type of(c) Average and standard deviation of the number of SCCP platform (SCCP Signaling and Diameter Signaling). signalling procedure.

messages and Diameter messages per IMSI per hour.

Figure 5: Signaling traffic time series for the observation period of December 2019.

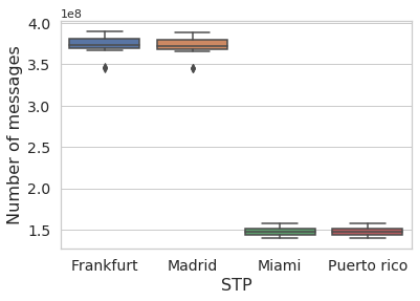

(a) SCCP global signaling.

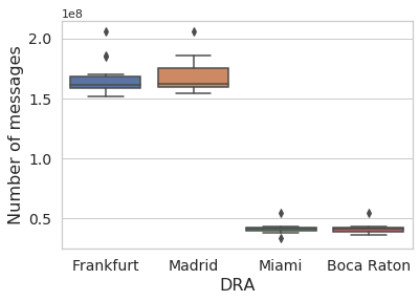

(b) Diameter signaling.

Figure 6: Signaling traffic load (total number of records) per infrastructure element per day for SCCP and Diameter. The boxplots capture the first two weeks in Dec. 2019.

messages. The latter, SAI, represents the highest fraction of MAP signaling traffic. According to the GSM standard definition, the Serving GPRS Support Node (SGSN) in the visited network triggers the authentication of subscriber procedure upon IMSI attach, location update or before data communication, explaining the large volume of SAI messages.

Figure $5 \mathrm{c}$ shows the average number of records per IMSI calculated over all the IMSIs we observe in each one-hour interval (continuous line) during the observation period, as well as the standard deviation of the number of records per IMSI calculated over all the IMSIs active in the same one hour interval (shaded area). We observe both the MAP procedures for $2 \mathrm{G} / 3 \mathrm{G}$ (red color) and the similar Diameter procedures for 4G/LTE (green color). While Diameter and MAP are different protocols, the underlying functional requirements (e.g., authenticating the user to set up a data communication) have many similarities in terms of the messages used for Diameter and the SS7 MAP protocol implementation. We note that the load in terms of average signaling records per IMSI is in the same order of magnitude (the continuous lines on the plot), regardless of the infrastructure the devices use; yet, there are significantly more messages for MAP, as Diameter is a more efficient protocol than MAP $[12,20]$.

We further investigate the traffic load on the signaling infrastructure, both for the SCCP (with STPs in Frankfurt, Madrid, Miami and Puerto Rico) and the Diameter signaling infrastructure (using DRAs in Frankfurt, Madrid, Miami and Boca Raton). Figure 6 shows the aggregated signaling traffic (number of messages) per infrastructure point per day over the same observation period. We note that the redundant

deployment of infrastructure points in each geographical area allows the IPX-P to load-balance the signaling traffic across them (e.g., for 4G between Frankfurt and Madrid in Europe and between Miami and Boca Raton in America). Signaling traffic in Europe through the signaling points in Frankfurt and Madrid is considerably higher than that flowing through the signaling points in America (Miami and Boca Raton/Puerto Rico). This is true for both the SCCP infrastructure (Fig. 6a) and the Diameter infrastructure (Fig. 6b). When comparing the two signaling services (i.e., SSCP and Diameter), we also note that the traffic volume in the SCCP signaling infrastructure is approximately twice larger than the traffic volume flowing through the Diameter signaling infrastructure. This proves the continued popularity of $2 \mathrm{G} / 3 \mathrm{G}$ services in the region served by the IPX-P.

\section{RELATED WORK}

The growing demand for global, mobile broadband access and a shift to all-IP-based services (from broadband last mile to VoIP) have brought new impetus to the old idea of the IPX, first proposed by the GSMA in 2007 to replace the traditional, bilateral-agreement model for international roaming [6]. Despite the continuous technical development by IPX-Ps and the related parties $[7,21,22]$ there has been few academic works on the topic. Takaaki [16] provides an early survey of IPX and its technical requirements, but there have been no in-depth analysis of IPX since due, in part, to its closed nature. Our work presents the first in-depth analysis of the IPX Network and the associated ecosystem.

\section{CONCLUSION}

In this paper, we provided both a qualitative and quantitative description of the IPX ecosystem using information collected from the one of the largest IPX providers. We believe that understanding the IPX network is cornerstone to understand and evolve the mobile Internet, and that it will become more relevant as new services emerge. For example, relying on IPX services, novel technologies such as eSIM that allow remote provisioning of mobile devices permit Global Mobile Network providers (e.g., Truphone) to emerge and offer novel connectivity options to end-users.

Volume 50 Issue 4, October 2020 


\section{REFERENCES}

[1] 2019. International Tourism Highlights, 2019 Edition. World Tourism Organization, Madrid, Spain. https://doi.org/10.18111/9789284421152

[2] Bernhard Ager, Nikolaos Chatzis, Anja Feldmann, Nadi Sarrar, Steve Uhlig, and Walter Willinger. 2012. Anatomy of a large European IXP. In Proceedings of the ACM Conference on Applications, technologies, architectures, and protocols for computer communication (SIGCOMM). 163-174.

[3] Mostafa Ammar. 2018. Ex Uno Pluria: The Service-Infrastructure Cycle, Ossification, and the Fragmentation of the Internet. SIGCOMM Comput. Commun. Rev. 48, 1 (April 2018), 56-63. https://doi.org/10. 1145/3211852.3211861

[4] GSM Association. [n. d.]. Operator Membership. ([n. d.]). Retrieved Nov 23, 2019 from https:/www.gsma.com/membership/ membership-types/operator-membership/

[5] GSM Association. 2012. IPX White Paper. (2012). Retrieved Dec 1, 2019 from https://www.gsma.com/iot/wp-content/uploads/2012/03/ ipxwp12.pdf

[6] GSM Association. 2013. IR.34-Guidelines for IPX Provider networks, Version 9.1. (2013). Retrieved Dec 29, 2019 from https://www.gsma. com/newsroom/wp-content/uploads/2013/05/IR.34-v9.1.pdf

[7] Rajesh Bhalla. 2012. Quality of service (qos) over network-tonetwork interfaces for ip interconnection of communication services. (2012). Retrieved Feb 4, 2020 from https://patents.google.com/patent/ US20120218924 [US Patent US20120218924A1].

[8] European Commission. 2015. New Rules on Roaming Charges and Open Internet. (2015). Retrieved Mar 6, 2018 from https://ec.europa.eu/digital-single-market/en/news/ new-rules-roaming-charges-and-open-internet

[9] European Commission. 2017. Roaming charges| What has the European Commission done so far? (2017). Retrieved Dec 29, 2019 from https://ec.europa.eu/digital-single-market/en/ roaming-charges-what-has-european-commission-done-so-far

[10] Jim Cowie. 2011. Building the Mobile Internet. https://archive.nanog. org/meetings/nanog51/presentations/Monday/cowie.pdf. (2011) [NANOG 51 Panel].

[11] Amsterdam Internet Exchange. [n. d.]. Connected networks, ams-ix. ([n. d.]). Retrieved Nov 15, 2019 from https://www.ams-ix.net/ams/ connected-networks

[12] Victor Fajardo, Jari Arkko, John A. Loughney, and Glen Zorn. 2012. Diameter Base Protocol. RFC 6733. (Oct. 2012). https://doi.org/10. 17487/RFC6733

[13] ROCCO HQ. 2019. ROCCO research: IPX networks vendor performance research for 2019 begins. (2019). Retrieved Nov 21, 2019 from https://www.roccoresearch.com/2019/06/19/ launch-ipx-networks-vendor-performance-research-2019/

[14] Geoff Huston. 2020. BGP in 2019. (2020). https://blog.apnic.net/2020/ 01/14/bgp-in-2019-the-bgp-table/

[15] Anna Maria Mandalari, Andra Lutu, Ana Custura, Ali Safari Khatouni, Ȯzgü Alay, Marcelo Bagnulo, Vaibhav Bajpai, Anna Brunstrom, Jörg Ott, Marco Mellia, et al. 2018. Experience: Implications of Roaming in Europe. In Proceedings of the 24th Annual International Conference on Mobile Computing and Networking (MobiCom). 179-189.

[16] Takaaki Moriya. 2013. Survey of IPX (IP eXchange) as an Emerging International Interconnection between Telecommunication Networks. IEICE Transactions on Communications E96.B (04 2013), 927-938. https: //doi.org/10.1587/transcom.E96.B.927

[17] Isabelle Paradis and Steve Heap. 2014. IPX Competitive analysis, Who offers what (sample). (2014). Retrieved Nov 21, 2019 from http: //www.hottelecom.com/reports/IPX-competitors-sample.pdf

[18] Hideyuki Sasaki. 2017. Peering culture can improve mobile Internet. (2017). Retrieved Jan 22, 2020 from https://blog.apnic.net/2017/05/12/ peering-culture-can-improve-mobile-internet/ [APNIC Blog Online].

[19] Kelsey Sheehy. 2019. Best International Cell Phone Plans 2019. (October 2019). Retrieved Feb 3, 2020 from https://www.nerdwallet.com/ blog/utilities/best-international-cell-phone-plans/

[20] Tina Tsou, Ruibing Hao, and Tom Taylor. 2013. Realm-Based Redirection In Diameter. RFC 7075. (Nov. 2013). https://doi.org/10.17487/ RFC7075

[21] Richard Xu. 2015. Method and Apparatus for Managing Communication Flow in an Inter-Network System. (2015). Retrieved Feb 4, 2020 from https://patents.google.com/patent/US20150222554 [US Patent US20150222554].

[22] Richard Xu, Hwan Jang Tang, and Ajay Joseph. 2014. Method and System For Hub Breakout Roaming. (2014). Retrieved Feb 4, 2020 from https://patents.google.com/patent/US20140169286 [US Patent US20140169286A1]. 


\section{A ACKNOWLEDGMENTS}

We are grateful to our reviewers for their insightful comments and suggestions. We would like to thank Francisco Jose Ballesteros Ruiz, Daniel Hidalgo Pazos and the rest of the team from Telefónica International Wholesale Services for their invaluable support collecting and analyzing the dataset from the IPX Provider. The work of Andra Lutu was supported by the EC H2020 Marie Curie Individual Fellowship 841315 (DICE).

\section{B ETHICAL CONSIDERATIONS}

Data collection and retention at network middle-boxes are in accordance with the terms and conditions of the IPX-P and the local regulations, and only with the specific purpose of providing and managing the IPX service. The terms also include data processing for monitoring and reporting as allowed usages of collected data. Data processing only extracts aggregated information and we do we not have access to any personally identifiable information. We nevertheless consulted with the Institutional Review Board (IRB) office at our institution who confirmed that no IRB review was necessary as the study relies on the analysis of de-identified data.

Table 1: List of active IPX Providers.

\begin{tabular}{|c|c|c|c|}
\hline IPX-P Name & BGP & Peering Policy & PoPs \\
\hline BICS & $\mathrm{X}$ & case-by-case & $120+$ \\
\hline BT & & IPX/GRX only & \\
\hline$C \& W$ & $\mathrm{X}$ & closed & \\
\hline China Mobile & & custom & 9 \\
\hline CITIC Telecom & & closed & $20+$ \\
\hline Comfone & & closed & 11 \\
\hline Deutsche Telekom & $\mathrm{X}$ & case-by-case & 30 \\
\hline Etisalat & $\mathrm{X}$ & case-by-case & 14 \\
\hline HGC & $\mathrm{X}$ & & \\
\hline iBasis & & & 100 \\
\hline MEO & $\mathrm{X}$ & open & \\
\hline MTT & $\mathrm{X}$ & open & \\
\hline MTX hub & & open & \\
\hline NTT & $\mathrm{X}$ & closed & \\
\hline Orange & $\mathrm{X}$ & & 250 \\
\hline OTE Globe & $\mathrm{X}$ & open & 21 \\
\hline PCCW Global & & case-by-case & \\
\hline Syniverse & $\mathrm{X}$ & case-by-case & \\
\hline TATA & $\mathrm{X}$ & case-by-case & $400+$ \\
\hline TDC A/S & $\mathrm{X}$ & case-by-case & \\
\hline Tele2 AB & $\mathrm{X}$ & selective & \\
\hline Telecom Italia & $\mathrm{X}$ & & 122 \\
\hline Telefonica & $\mathrm{X}$ & case-by-case & $100+$ \\
\hline Telekom Austria & $\mathrm{X}$ & & 148 \\
\hline Telenor & $\mathrm{X}$ & closed & 13 \\
\hline TeliaSonera & $\mathrm{X}$ & & $300+$ \\
\hline Telstra & $\mathrm{X}$ & open & 36 \\
\hline TNS & $\mathrm{X}$ & open & 125 \\
\hline Vodafone & $\mathrm{X}$ & case-by-case & 273 \\
\hline
\end{tabular}

ACM SIGCOMM Computer Communication Review

\section{IPX PROVIDERS}

We compiled a list of 29 IPX-Ps that, at the time of writing, interconnect to form the IPX Network. Table 1 lists the IPX-Ps we identified as active in the IPX Network, their (public) peering policy, and number of PoPs. We built the list of IPX-Ps and the information we show in the table by manually exploring the peering dataset. Specifically, at AMS-IX we found 27 customers for the mobile peering service with IPX/GRX tags [11]. In addition, we found 24 of the total 27 IPX-Ps peering at AMS-IX also present at Equinix IXPs. According to GSMA, the majority of IPX-Ps connect to these two IXPs [6]. Additionally, we checked the top 10 largest IXPs and some global IXPs and found no additional IXPs offering the mobile peering service. We complete this list with two additional IPX-Ps we observe in the internal list of peers from the operational IPX-P, thus bringing the total number of players in the IPX Network to 29, which we list in Table 1. We confirm this list and the IPX-Ps' identities by checking several commercial market surveys and reports about IPX-Ps from diverse parties [10,13, 17]. We verify that these IPX-Ps appear in the routing dataset as active peers of the IPX-P providing us the routing table snapshot (see Section 3.2). We find a set of 23 different ASes, which we mark in Table 1 in the "BGP" column. We also find that among the set of currently active IPX-Ps, there are several which focus on interconnecting SPs within a specific region (e.g., Telin Indonesia, SAP). We do not include these in our analysis, but instead focus on the list we show in Table 1. The number of PoPs in Table 1 indicates the number of locations where an IPX-P can cross-connect with SPs, giving insight into its world-wide geographical footprint. The average number of PoPs among the 18 IPXs publicly disclosing this information is 116 .

Table 2: Comparison between IPX and public Internet ${ }^{6}$

\begin{tabular}{|c|c|c|}
\hline Subject & $\begin{array}{l}\text { IPX } \\
\text { Ecosys- } \\
\text { tem }\end{array}$ & Public Internet \\
\hline Type of interconnections & Full-mesh & $\begin{array}{l}\text { Transit, Peering, } \\
\text { Hybrid }\end{array}$ \\
\hline Length of IP prefixes & $/ 32$ & $/ 24$ \\
\hline Size of BGP routing tables & 10,418 & 814,000 \\
\hline Avg. length of the AS-Path & 2 & 5.6 \\
\hline Transit ASes & None & 9,600 \\
\hline Support for IPv6 & Yes & Yes \\
\hline BGP Communities & Yes & Yes \\
\hline
\end{tabular}

In Table 2 we summarize the main characteristics of the IPX Ecosystem, in comparison with the public Internet. As previously stated, the IPX ecosystem includes the IPX-Ps we

${ }^{6}$ Statistics of the public Internet are from APNIC's annual report for BGP [14]. 
list in in Table 1 connecting in a full mesh, as well as their customers. This is significantly smaller than the public Internet,

${ }^{7}$ Check the CIDR Report for updates on routing metrics for the public Internet: https://www.cidr-report.org/as2.0/ where we see a total of over 65,000 ASes interconnecting at the interdomain level, through various types of relationships (including transit, peering, paid peering, hybrid). ${ }^{7}$ 